\title{
Intratracheal aerosolization of viral vectors to newborn pig airways
}

\author{
Ashley L Cooney ${ }^{1}$ \& Patrick L Sinn*,1
}

\section{ABSTRACT}

Gene therapy for airway diseases requires efficient delivery of nucleic acids to the airways. In small animal models, gene delivery reagents are commonly delivered as a bolus dose. However, large animal models are often more relevant for the transition from preclinical studies to human trials. Aerosolizing viral vectors to the lungs of large animals can maximize anatomical distribution. Here, we describe a technique for aerosolization of viral vectors to the airways of newborn pigs. Briefly, a pig is anesthetized and intubated with an endotracheal tube, and a microsprayer is passed through the endotracheal tube. A fine mist is then sprayed into the distal trachea. Widespread and uniform distribution of transgene expression is critical for developing successful lung gene therapy treatments.

\section{METHOD SUMMARY}

Historically, achieving uniform distribution of a gene therapy reagent in the lungs has been challenging. Here, we describe an aerosolizing technique which can be used to achieve homogenous expression of a viral vector in newborn pig lungs. Briefly, pigs are sedated and intubated with an endotracheal tube, and a microsprayer is used to aerosolize a viral vector which results in its widespread distribution in the lungs.

\section{KEYWORDS}

gene therapy $\cdot$ gene transfer - intubation - microsprayer $\cdot$ porcine - viral vector delivery

${ }^{1}$ Stead Family Department of Pediatrics, Pappajohn Biomedical Institute, \& Center for Gene Therapy, The University of lowa, lowa City, IA 52242, USA ; *Author for correspondence:patrick-sinn@uiowa.edu

BioTechniques 68: 235-239 (May 2020) 10.2144/btn-2019-0150
Gene transfer to the lung holds great potential for treating genetic diseases such as cystic fibrosis (CF) or alpha1-antitrypsin deficiency. However, developing successful approaches to delivering genes of interest to the airways has been challenging. Animal models play a major role in driving innovation of viral vector design and delivery strategies to the intrapulmonary airways $[1,2]$. Indeed, we and others have developed methods to overcome many gene delivery hurdles using large animal models. Examples of delivery challenges have been reviewed previously [3-6]. Using the newborn pig as a large animal model, we have refined a protocol to achieve widespread airway distribution following intratracheal aerosol delivery.

Here, we demonstrate how to achieve efficient viral vector delivery to a newborn pig lung through aerosolization. Conceptually, topical delivery of a vector encoding a therapeutic transgene to the lung is simple. However, in practice, achieving efficient delivery is a challenge. Important considerations include the choice of viral vector, appropriate vehicle for the vector and aerosolization method. In general, devices for generating an airborne vector fall into three categories: aerosolizing catheters, atomizers and nebulizers. All of these devices convert liquids into particles small enough to be respired. Aerosolizing catheters convert liquids into particles at the point of expulsion. For these studies, we used a syringe-mounted aerosolizing catheter termed a microsprayer. We selected a microsprayer as our aerosolization device in part for its ease of use and also because of its ability to effectively aerosolize a viral vector in a particle size that can reach all areas of the lung. The microsprayer works by generating an aerosol at its tip that results from the force generated by depressing a syringe plunger. We validated this delivery method for both adenoviral (Ad)- and adenoassociated virus-based viral vectors $[7,8]$. A microsprayer may be less suited to delivering enveloped viral vectors (e.g., lenti- virus) due to resultant drops in functional titer (AL Cooney and PL Sinn, unpublished observation), presumably as a result of shear stress on the viral particles.

We previously validated this technique for delivering gene transfer vectors to pig lungs and showed widespread and uniform airway distribution following delivery of an $\mathrm{Ad}$ vector expressing green fluorescent protein (GFP) [7]. To assess transduction, animals were humanely euthanized, lungs were resected 5 days postdelivery and all six lung lobes were separated into 2-4 segments (resected pieces varied in size depending on the lobe, but ranged from 0.5 to $8 \mathrm{~cm}^{3}$ ). From each segment, tissue was designated for DNA or mRNA isolation and transduction was quantified by real-time PCR to detect the GFP sequence. GFP-positive cells were counted and graphed as a function of airway diameter. Remarkably, we achieved abundant gene transfer throughout large and small airways. We observed a variety of transduced cell types, including surface epithelium (ciliated, nonciliated), basal cells and submucosal gland cells [7]. Droplet particles can reach the most distal regions of the lung, expressing a transgene in epithelial cells of both the cartilaginous and noncartilaginous airways.

Using laser diffraction, we quantified the size of the microsprayer-produced droplets and obtained consistent measurements of 15-16 $\mu \mathrm{m}$. Smaller particle sizes may be possible using aerosolizing catheter devices that utilize pressurized delivery through compressed air, which may permit the generation of particles as small as $4-8 \mu \mathrm{m}$. Such a device was used to aerosolize helperdependent adenovirus vectors to rabbit airways $[9,10]$ and Sendai virus vectors to sheep [11]. Atomizers are a subclass of aerosolizing catheters that deliver larger particles ( 30-90 $\mu \mathrm{m}$ in diameter). Our group observed that this type of atomizer is an effective delivery device for multiple viral vectors including lentiviral vectors, particularly when formulated with a visco- 
elastic material such as methylcellulose [12]. Nebulizers first convert the liquid into a mist that is passively inhaled. Using this strategy, a plasmid-based vector was delivered to the airways of CF patients in a Phase IIB gene therapy trial [13]. Nebulization requires a large volume of concentrated material, so is the least economic delivery strategy for viral vectors.

Prior to developing this protocol, we tested multiple different delivery methods in 3-4-week-old pigs. For example, we delivered vector to a localized lung lobe using a pediatric bronchoscope and compared a bolus liquid dose with a drug infusion balloon [14]. Additionally, we tested an atomizer [15] and a pressurized aerosolizing catheter [AL Cooney and PL Sinn, Unpublished Data]. The pressurized aerosolizing catheter delivery was effective but required extra equipment and the pressurized delivery occasionally resulted in injury to pig tracheas. Based on ease of use and reproducibility, we now routinely opt for the syringe-mounted microsprayer for delivery of encapsidated viral vectors such as Ad and adeno-associated viral vectors. However, the atomizer gives the most comparable lung expression to the microsprayer and does not require an endotracheal (ET) tube. Although our focus has been on developing a delivery method for efficient lung gene transfer to correct CF, this method could be adapted for other applications. The aerosolization device and droplet size may play important roles in the efficiency and distribution of vectormediated transgene expression. Here, we focus on the procedure of intubation in newborn pigs and passing a microsprayer through an ET tube to deliver the vector.

\section{MATERIALS \& METHODS}

Prepare the procedure space \& vector delivery methods

Please see Supplemental Protocol and Methods for stepwise protocol and Supplementary Table 1 for a list of materials used. Begin by setting up a heating pad covered by a disposable underpad, the pulse oximeter and rectal thermometer coated with lubricating jelly. Next, pass the microsprayer through a 2.0-mm ET tube and mark the base of the microsprayer when the tip exits the ET tube by $1 \mathrm{~mm}$. Since ET tubes may vary slightly in length, it is important to confirm how far to pass the microsprayer through each ET tube. After the microsprayer has been marked, remove the microsprayer from the ET tube; attach a 1- or 3-ml syringe filled with saline to the microsprayer and perform a test spray. This serves two purposes: it confirms that the microsprayer is not clogged and it allows the user to practice an appropriate force to generate a mist, as too little pressure results in a stream instead of a spray. Following completion of the test spray, the microsprayer can be assembled with a syringe containing 1-2 $\mathrm{ml}$ of viral vector and set aside for delivery. Next, prepare the ET tube for intubation by inserting a stylet and precoating the end of the ET tube with lubricating jelly. At last, set up the isoflurane regulator to prepare for sedation. To do this, connect the $\mathrm{O}_{2}$ tank with a pressure regulator and flowmeter to the vaporizer, connect the vaporizer through tubing to deliver the isoflurane through an anesthesia mask, and an anesthesia gas filter canister to collect the waste anesthesia gas from the operating room environment. Arrange the procedure area with a 4" elongated blade laryngoscope, the microsprayer with syringe containing viral vector and the ET tube containing a stylet.

\section{Sedate pigs}

Ensure the pig has fasted prior to sedation to prevent aspiration during delivery. Monitoring will be necessary during the procedure; begin by wrapping a $\mathrm{SpO}_{2}$ sensor to the pig's hind leg to ensure the readings register on the pulse oximeter and record a preanesthetic reading. Next, turn on the $\mathrm{O}_{2}$ tank (with the flowmeter set to $2 \mathrm{l} / \mathrm{min}$ ) and the isoflurane vaporizer (recommended range $2-4 \%$ ) and place the nose cone over the pig's snout until the pig is sedated. This will take approximately $4-5 \mathrm{~min}$ but the time will vary depending on the age and weight of the animal. Begin by holding the pig during the initial stages of anesthesia. Once the pig is sedated, lay it on the prepared procedure space (underpad over a heating pad). Confirm anesthesia by testing the pedal reflex. Record rectal temperature, respiratory rate and heart rate at this point and continue with procedural monitoring every $15 \mathrm{~min}$ throughout sedation and recovery.

\section{Intubate sedated pigs with ET tube}

Once the pig has been sedated, double check that the ET tube lined with a stylet has been lightly coated with lubricating jelly to facil- itate intubation. Remove the anesthesia mask from the pig and turn off the flow of isoflurane. Begin the intubation process by laying the pig supine on the procedure space and visualizing the larynx using a laryngoscope. Once visual confirmation of the larynx is achieved, pass the ET tube lined with stylet through the vocal folds of the larynx and into the trachea (Supplementary Movie 1). If properly intubated, the $\mathrm{SpO}_{2}$ levels will start to decline due to the airway obstruction by the ET tube. Note that the exact tracheal region reached by the ET tube will vary depending on the size of the animal. Placement can vary from just beyond the larynx for larger animals $(\sim 3-4 \mathrm{~kg})$ to near the carina for smaller animals $(\sim 0.8-1 \mathrm{~kg})$. In small animals, there is a risk of one side intubation; trimming 3-5 cm from the ET tube may be warranted. After intubation, remove the stylet from the ET tube.

\section{Aerosolize the viral vector using the microsprayer}

Pass the microsprayer (connected to viral vector-containing syringe) through the ET tube until it reaches the mark at the base of the microsprayer. Spray the solution intratracheally by pressing the syringe plunger with firm and consistent force to generate a mist; this will take approximately $3-4 \mathrm{~s}$ (the recommended volume to deliver is $\sim 1 \mathrm{ml} /$ $\mathrm{kg}$ ). A postspray 'air chaser' of approximately $500 \mu \mathrm{l}$ will help to ensure complete delivery of vector from the syringe and nozzle. Gently remove the ET tube and microsprayer from the intratracheal intubation at the same time. Successful delivery will typically result in audible crackles as the pig breathes.

\section{Monitor pigs as they come out of} sedation

Apnea is a common response to intubation in newborn pigs and sporadic breathing may last 2-3 min. Gentle chest compressions can help to facilitate normal breathing. Monitor $\mathrm{SpO}_{2}$ levels until they return to $95-100 \%$, then remove the $\mathrm{SpO}_{2}$ sensor from the pig's hind leg, continuing with postprocedural monitoring every 15 min until the pig is alert, sternal and walking. Typically, pigs will recover within 15 min.

\section{RESULTS \& DISCUSSION}

There are multiple strategies to confirm successful intubation and vector delivery. 
When first learning this technique, it is helpful to practice on a euthanized pig; after intubation, the trachea can be visualized via dissection and successful intubation can be confirmed immediately. When appropriate in our studies, we employ computerized tomography (CT) to confirm placement of the ET tube in the trachea (Figure 1) and the distribution pattern of delivered material throughout the lung (Figure 2). The pre and postdelivery CT images (Figure 2A \& B, respectively) confirm delivery of material to the pulmonary airways. 'Cloudy' CT images result from the aerosolized liquid and indicate a successful delivery (Figure 2B, black arrows). Contrast agents are not necessary. In past experiments, we used $x$-ray imaging to confirm airway catheter placement in rabbits [16], as $\mathrm{x}$-ray is less expensive and more accessible than CT imaging. Alternatively, fiberoptic micro-camera technologies and single-use flexible video bronchoscopes are potentially adaptable to validating ET tube placement.

To confirm successful viral vectormediated gene transfer to the airways, we use viral vectors that express standard reporter genes such as GFP. In the example shown in Figure 3, an Ad vector expressing GFP formulated with $0.1 \%$ lysophosphatidylcholine was aerosolized to the airways of a newborn pig. Lysophosphatidylcholine is a natural airway surfactant that will transiently disrupt tight junctions to allow Ad to access its basolateral receptor [17]. Five days later, we collected the lungs and used low-power fluorescence microscopy to examine the fresh, unfixed tissue. We observed abundant GFP expression spread uniformly throughout the lung lobes (Figure 3). Low-power epifluorescent and bright-field views of the right middle lobe are shown (Figures $3 \mathrm{~A} \& \mathrm{~B}$ ).

Low-power fluorescent images of whole tissue will typically reveal alveolar expression. Gene expression in conducting airways can be confirmed by manual dissection. As we previously reported [8], tissue should be fixed, sectioned and mounted on slides. Highpower microscopy can be used to confirm the number of transduced epithelial cells in the conducting airways. In this example from our Ad vector expressing GFP experiment (Figure 4), parenchymal tissue from transduced pig lungs was removed and airway branches were imaged using a fluorescent dissecting microscope. GFP expression indicates widespread distribution in the conducting airways. In this low-power image, GFP expression appears fainter in the large (cartilaginous) airways because the thicker tissue masks the epithelial signal on the luminal surface.

Widespread airway distribution of a viral vector would help to ensure the success of a gene therapy approach for treating pulmonary diseases. Here, we describe an aerosolization technique that leads to whole-lung expression in large and small pig airways. We outline the steps for sedating a pig, intubating with an ET tube and aerosolizing a viral vector through the microsprayer aerosolization device. This technique is important as a preclinical approach to testing viral vector efficacy and is most applicable to newborn pigs $(1 \mathrm{~kg})$ that are too small to be intubated with standard pediatric bronchoscopes.

There are multiple critical steps in this procedure. The most critical step is ensuring correct placement of the ET tube in the trachea and not the esophagus. The intubation technique can be improved with practice, beginning with intubating a euthanized pig and immediately checking for proper ET tube placement by dissecting a tracheal window. Multiple examples of verifying ET tube placement are described in the Results; however, with practice, intubation will become reliable and routine. If available, a CT scan

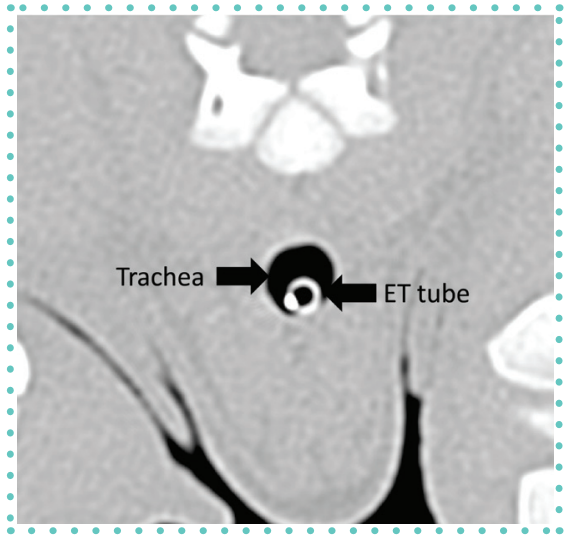

Figure 1. Computerized tomography confirmation of tracheal placement of endotracheal tube. While under isoflurane anesthesia, a newborn pig was intubated with an ET tube. The pig was subsequently imaged using a computerized tomography scanner. Proper placement of the ET tube was confirmed in the trachea (black arrows).

ET: Endotracheal.

can verify ET tube placement before vector delivery; pre- and postdelivery CT imaging is also a potential strategy to determine general deposition patterns. The second critical factor is that the microsprayer tip should just exit the ET tube. If the microsprayer tip does not extend past the end of the ET tube, the aerosolization will pool within the ET tube and exit as a bolus dose. If the microsprayer tip extends too far beyond the ET tube, there is a risk of the microsprayer tip puncturing the wall of the trachea and causing a pneumothorax. Using the protocol described, we have never

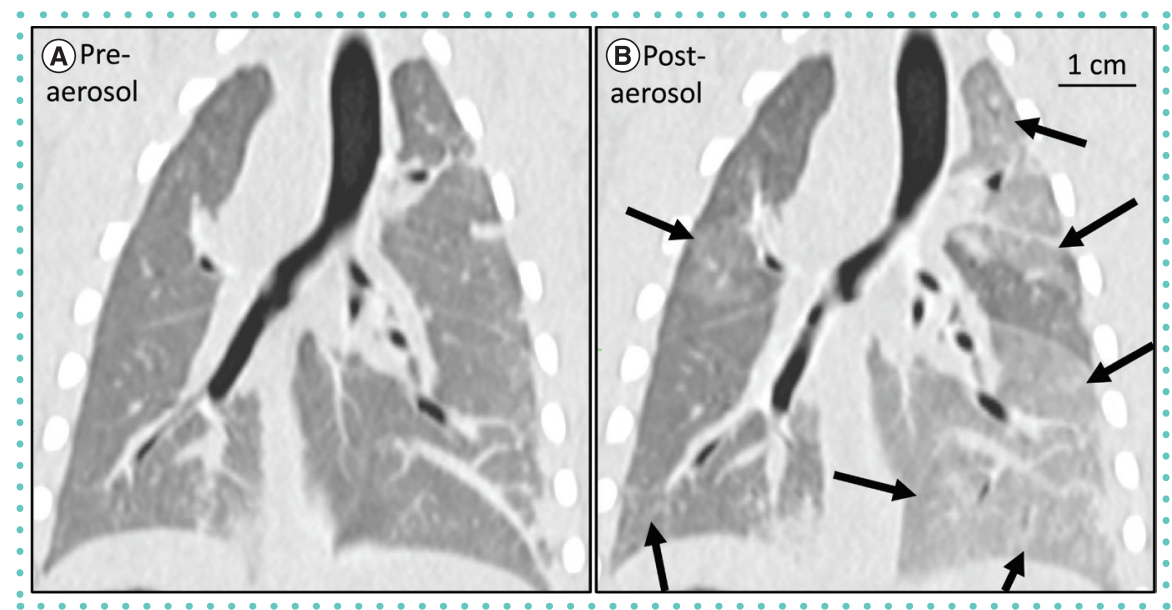

Figure 2. Example of widespread aerosol delivery confirmation. Computerized tomography images of pig lungs were collected (A) pre and (B) postvector aerosolization. (A) Following intubation, but before vector delivery, the lungs were imaged. (B) Adenoviral-green fluorescent protein formulated with $0.1 \%$ lysophosphatidylcholine was aerosolized through the microsprayer and the pig was imaged again. The resultant 'cloudy' appearance (black arrows) is indicative of successful aerosol delivery. 


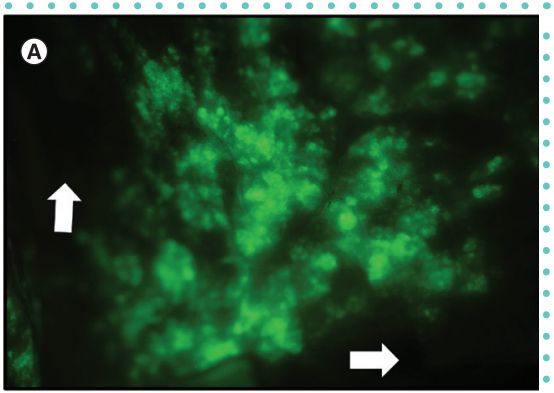

B

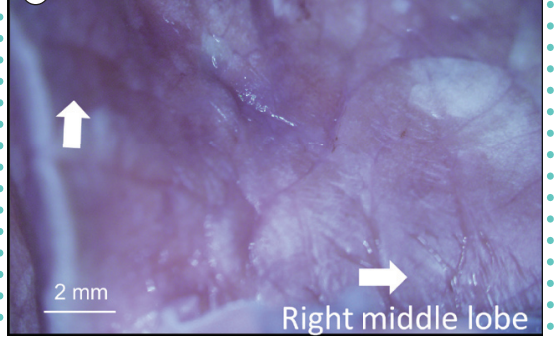

Figure 3. Representative images from a pig transduced with adenoviral-green fluorescent protein, showing high levels of gene transfer. Adenoviral-GFP formulated with $0.1 \%$ lysophosphatidylcholine was aerosolized intratracheally to newborn pigs. Five days later, lungs were collected and GFP expression was visualized by fluorescence microscopy of fresh unfixed tissue. Fluorescent (A) and bright-field (B) images of the right middle lobe were captured using a fluorescent dissecting microscope. White arrows indicate untransduced regions of the lobe. GFP: Green fluorescent protein.

observed a pneumothorax or other serious complication. However, if the animal displays an extended period (>20 $\mathrm{min}$ ) of labored breathing or signs of distress, it should be euthanized for autopsy. Finally, an appropriate plane of anesthesia will facilitate successful delivery. Newborn pigs typically begin to recover quickly (<10 $\mathrm{min}$ ) from isoflurane so the procedure should be performed with care for time management. If a pig is too lightly sedated, it may kick or clench its jaw. Conversely, too much sedation will needlessly prolong the procedure and recovery time.

There are multiple options for vector delivery to the airways and this procedure may be adapted for different devices. When deciding which aerosolizing device to use for any application, it is helpful to know how each device works. In mice, a bolus dose results in high levels of expression in the lung [12,18]; however, to achieve these levels as a bolus dose in a large animal, a small region or specific lobe would need to be targeted. Such targeting was previously described in rats [19]. Delivery to a

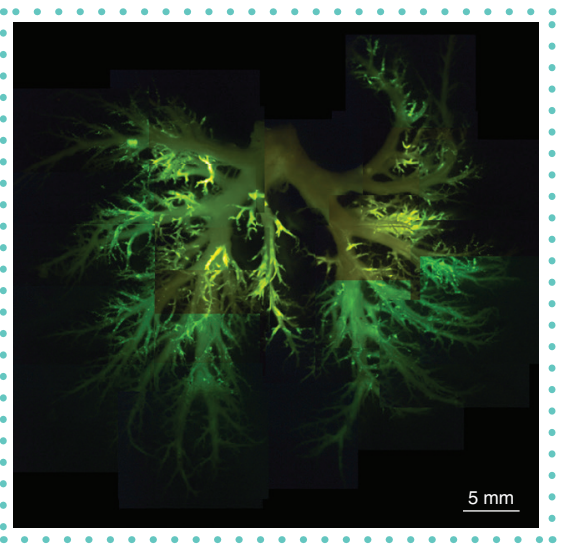

Figure 4. Gene transfer in conducting airways. Five days after aerosol delivery of adenoviralGFP, lungs were collected. The parenchymal tissue was removed through manual dissection. As shown by the photo montage, GFP-positive expression in the conducting airways was confirmed using a fluorescent dissecting microscope. GFP: Green fluorescent protein.

small region within the lung requires visual guidance by a bronchoscope. We validated successful gene delivery to 4-week-old pig airways using a bronchoscope lined with a PE20 catheter [14]; that delivery method is limited to a specific region of the lung, which may be useful for particular applications. An atomizer is a good choice for whole lung viral vector delivery [15]. The atomizer results in widespread delivery at a particle size of $30-90 \mu \mathrm{m}$ and is effective at delivering lentiviral vectors formulated with viscoelastic gel $[12,15]$. The atomizer we used previously [15] has a bulb-shaped tip that can be challenging to pass through the vocal folds of a newborn pig. A pressurized aerosolizing catheter leads to high levels of gene transfer by a lentivirus in sheep lungs [11] and also has been used to deliver liposomal formulations [20]. A recent study in newborn pigs evaluating the use of a nebulizer to aerosolize nonviral particles reported heterogeneous expression among lung lobes [21]. This suggests that an aerosolizing device generating finer particles delivered directly into the trachea could result in more homogenous gene expression patterns throughout the airway tree. It is important to note that the microsprayer used here, and the pressurized aerosolizing catheter screened previously (see Table of Materials), are no longer available for purchase. Suitable substitutions will need to be identified.
This method requires skill in the intubation technique. Practice and hands-on training will facilitate success. Alternate methods such as nasal instillation or nebulization are less invasive, do not require full sedation, and are easier to learn. However, viral vectors delivered via nasal instillation may not reach to the distal airways in large animal models and nebulization requires a volume of viral vector that is often cost prohibitive. Using intratracheal aerosolization to newborn pig airways, we can rapidly and efficiently deliver vector directly to the large and small airways. From practical experience, we know that $1 \mathrm{ml} / \mathrm{kg}$ of Ad vector ( $10^{10}$ transducing units $/ \mathrm{kg}$ ) is sufficient to reach all regions from the trachea to the small bronchioles in newborn pigs.

\section{FUTURE PERSPECTIVE}

With the development of novel gene addition and gene editing tools for airway disease, consideration must be given to how these reagents will be delivered to the airways of animal models. A potential hurdle for testing the success of a lung gene therapy reagent is the identification of a delivery method that distributes to all areas of the lung. A method that results in homogeneous delivery throughout the entire lung could be applicable to either viral or nonviral vectors. A method that achieves widespread lung distribution could expedite clinical development of new therapeutic reagents with higher success rates.

\section{SUPPLEMENTARY DATA}

To view the supplementary data that accompany this paper please visit the journal website at: www.future-science. com/doi/suppl/10.2144/btn-2019-0150

\section{AUTHOR CONTRIBUTIONS}

AL Cooney: experimental design, performing experiments, data analysis and manuscript preparation. PL Sinn: experimental design, performing experiments, data analysis and manuscript preparation.

\section{ACKNOWLEDGMENTS}

We thank C Brommel for aiding with the airway dissection and RV Rada with the supplemental movie. We thank the University of lowa Office of Animal Resources and the animal caretakers. We thank the Viral Vector Core for vector production. 


\section{FINANCIAL \& COMPETING}

\section{INTERESTS DISCLOSURE}

This work was supported by the National Institutes of Health (NIH P01 HL-51670, NIH P01 HL-091842, NIH R01 HL-133089 and NIH R01 HL-105821), the Center for Gene Therapy of Cystic Fibrosis (NIH P30 DK-054759) and the Cystic Fibrosis Foundation (SINN19XXO and COONEY18F0). The authors have no other relevant affiliations or financial involvement with any organization or entity with a financial interest in or financial conflict with the subject matter or materials discussed in the manuscript apart from those disclosed.

No writing assistance was utilized in the production of this manuscript.

\section{ETHICAL CONDUCT OF RESEARCH}

All animal experiments performed following this protocol must be approved by the respective Institutional Animal Care and Use Committee (IACUC). All procedures described here were approved by the University of lowa IACUC.

\section{OPEN ACCESS}

This work is licensed under the AttributionNonCommercial-NoDerivatives 4.0 Unported License. To view a copy of this license, visit http://creativecommons.org/licenses/ by-nc-nd/4.0/

\section{REFERENCES}

Papers of special note have been highlighted as: • of interest; • o of considerable interest

1. Mclachlan G, Baker A, Tennant $P$ et al. Optimizing aerosol gene delivery and expression in the ovine lung. $\mathrm{Mol}$. Ther. 15(2), 348-354 (2007).

2. Davidson H, Mclachlan G, Wilson A et al. Human-specific cystic fibrosis transmembrane conductance regulator antibodies detect in vivo gene transfer to ovine airways. Am. J. Respir. Cell Mol. Biol. 35(1), 72-83 (2006).

3. Koehler DR, Hitt MM, Hu J. Challenges and strategies for cystic fibrosis lung gene therapy. Mol. Ther. 4(2), 84-91 (2001).

4. Oakland M, Sinn PL, Mccray PB Jr. Advances in cell and gene-based therapies for cystic fibrosis lung disease. Mol. Ther. 20(6), 1108-1115 (2012)

5. Donnelley M, Parsons DW. Gene therapy for cystic fibrosis lung disease: overcoming the barriers to translation to the clinic. Front. Pharmacol. 9, 1381 (2018).

6. Cooney AL, MccrayPBJr, Sinn PL. Cystic fibrosis gene therapy: looking back, looking forward. Genes Basel) 9(11), 538; 1-23 (2018).

7. Cooney AL, Singh BK, Loza LM et al. Widespread airway distribution and short-term phenotypic correction of cystic fibrosis pigs following aerosol delivery of piggyBac/adenovirus. Nucleic Acids Res. 46(18), 9591-9600 (2018).

- Description of cell types transduced and quantification of cell transduction throughout the lung following aerosolization using the technique described in this manuscript.

8. Cooney AL, Thornell IM, Singh BK et al. Novel AAV-mediated gene delivery system corrects CFTR function in pigs. Am. J. Respir. Cell Mol. Biol. doi:10.1165/ rcmb 2019-00060C (2019) (Epub ahead of print).

Aerosolization using the microsprayer resulted in phenotypic correction in a large animal model for cystic fibrosis.

9. Koehler DR, Martin B, Corey M et al. Readministration of helper-dependent adenovirus to mouse lung. Gen Ther. 13(9), 773-780 (2006)

10. Koehler DR, Frndova $\mathrm{H}$, Leung $\mathrm{K}$ et al. Aerosol delivery of an enhanced helper-dependent adenovirus formulation to rabbit lung using an intratracheal catheter. J. Gene Med. 7(11), 1409-1420 (2005)

11. Griesenbach U, Mclachlan G, Owaki T et al. Validation of recombinant Sendai virus in a non-natural host model. Gene Ther 18(2), 182-188(2011).
12. Sinn PL, Shah AJ, Donovan MD, Mccray PB Jr. Viscoelastic gel formulations enhance airway epithelia gene transfer with viral vectors. Am. J. Respir. Cell Mol. Biol. 32(5), 404-410 (2005).

13. Alton EW, Boyd AC, Cheng SH et al. A randomised double-blind, placebo-controlled Phase IIB clinical trial of repeated application of gene therapy in patients with cystic fibrosis. Thorax 68(11), 1075-1077 (2013).

14. Sinn $\mathrm{PL}$, Cooney $\mathrm{AL}$, Oakland $\mathrm{M}$ et al. Lentiviral vector gene transfer to porcine airways. Mol. Ther. Nucleic Acids 1, e56 (2012)

- An alternative delivery method to the lung using the pig model.

15. Cooney AL, Abou Alaiwa MH, Shah VS et al. Lentiviral-mediated phenotypic correction of cystic fibrosis pigs. JCl Insight 1(14), pii: 88730 (2016).

Use of an atomizer to deliver a viral vector to a cystic fibrosis pig model and demonstration of phenotypic correction.

16. Sinn PL, Penisten AK, Burnight ER et al. Gene transfer to respiratory epithelia with lentivirus pseudotyped with Jaagsiekte sheep retrovirus envelope glycoprotein Hum. Gene Ther. 16(4), 479-488 (2005).

17. Cmielewski P, Anson DS, Parsons DW. Lysophosphatidylcholine as an adjuvant for lentiviral vector mediated gene transfer to airway epithelium: effect of acyl chain length. Respir. Res. 11, 84 (2010).

- Highlights the importance of using lysophosphatidylcholine to disrupt tight junctions to improve receptor access.

18. Wilson AA, Murphy GJ, Hamakawa $\mathrm{H}$ et al. Amelioration of emphysema in mice through lentiviral transduction mice through lentiviral transductic Invest. 120(1), 379-389 (2010).

19. Mcintyre C, Donnelley M, Rout-Pitt N, Parsons D. Lobe-specific gene vector delivery to rat lungs using a miniature bronchoscope. Hum. Gene Ther. Methods 29(5), 228-235 (2018).

Describes an aerosolized delivery using a miniature bronchoscope to a small animal model.

20. Gaspar MM, Gobbo O, Ehrhardt C. Generation of liposome aerosols with the Aeroneb Pro and the AeroProbe nebulizers. J. Liposome Res. 20(1), 55-61 (2010).

21. Caballero I, Riou $M$, Hacquin $O$ et al. Tetrafunctional block copolymers promote lung gene transfer in newborn piglets. Mol. Ther. Nucleic Acids 16, 186-193 (2019). 